\title{
Dynamic Error of Heat Measurement in Transient
}

\author{
Fang Lide, Li Jinhai, Cao Suosheng, Zhu Yan, and Kong Xiangjie \\ The Institute of Quality and Technology Supervising, Hebei University, Baoding, China, \\ 071051 \\ Leed_amy@yahoo.com.cn
}

\begin{abstract}
According to EN1434 (European Standard) and OIML R-75(International Organization of Legal Metrology), there are two methods in heat measurement, and they are all steady-state methods of test, using them in transient, obvious error will be produced, so some accurately measuring functions should be seeking. In a previous published paper of the author, a transient function relationship of heat quantity and time is deduced, and the validity of this function is proved through experimentation, also it is simplified reasonably, so it can be used in variable flow rate heating system. In this study, a comparison with steady-state method and dynamic method is presented, the errors exist in the steady state method are analyzed. A conclusion is improved that the steady-state methods used in variable flow heating system will result in appreciable errors, the errors only exist in transient, when system reach steady state, the errors disappear, moreover, the transient time is long in heating system, it is at least 30 minutes, so it is necessary to take some measures to correct them, however, study showed that the error can be ignored when the flow rate step change is less than $5 \mathrm{~kg} / \mathrm{h}$.
\end{abstract}

Keywords: Dynamic heat meter, variable flow heating system, step change, flux.

\section{Introduction}

The accuracy of measurement model is a continuous goal in measurement instrument designing, moreover, in last decades saving the energy has become an important issue, due to environmental protection and economical reasons. In every year a significant part of the energy is utilized for heating. So the primary concern of heating services today is to accurately measure and charge the heat consumption.

According to EN1434 (European Standard) and OIML R-75(International Organization of Legal Metrology), there are two methods in heat measurement, and they are all steady-state methods of test, Theory give the two methods as

$$
\begin{aligned}
& Q=\int_{\tau_{1}}^{\tau_{2}} G \Delta h d \tau \\
& Q=\int_{v_{1}}^{v_{2}} k \Delta \theta d V
\end{aligned}
$$


where: $Q$ is the quantity of heat given up; $G$ is the mass flow rate of the heat-conveying liquid passing through the heat meter; $\Delta h$ is the difference between the specific enthalpies of the heat-conveying liquid at the flow and return temperatures of the heat-exchange circuit; $t$ is time; $V$ is the volume of liquid passed; $k$ called the heat coefficient, is a function of the properties of the heat-conveying liquid at the relevant temperatures and pressure; $\Delta \theta$ is the temperature difference between the flow and return of the heat exchange circuit[1,2].

Usually, equation (1) called enthalpy difference method and equation (2) called $\mathrm{K}$ coefficient method; they are widely used in all kinds of heat meters [3-8].

Howeve $r$, when the flow rate of system have a step change, heat dissipating capacity is a function of flow rate, temperature and time, and, if the transient time interval between two steady states is long enough, huge error would be produced by using steady equation (1) or (2).

During the author study on a new heating measurement and control system, a function relationship of heat quantity and time is deduced, and the validity of the function is proved through experimentation, and it is simplified reasonably [9], so it can be used in variable flow heating system, this article presented a comparison of state heat measurement method and dynamic heat measurement method.

\section{Principle of Dynamic Heat Meter}

When system flux generated step change, the author put forward a "level-move" presumption of temperature distribution curve in radiator during transient. On the basis of the presumption, the function of heat quantity and time is deduced, and validity of the function is proved through experimentation, the relativity error of the function was within $3 \%$. The dynamic heat measurement function in transient after flux changing to large can be seen as Equation (3), and the opposite function can be expressed as equation (4) $[9,10,11,12]$.

$$
\begin{aligned}
& Q_{0-1}=c G_{0}\left(t_{g}-t_{n}\right)\left(1-e^{-b_{0} F_{C}}\right)- \\
& \frac{\left(t_{g}-t_{n}\right) \cdot\left(K_{0}+\left(K_{1}-K_{0}\right) \frac{\tau}{\tau_{s 1}}\right)}{b_{0}}\left(e^{-b_{0}\left(F_{C}-\frac{\tau}{\tau_{s 1}} \cdot a_{0-1}\right)}-e^{-b_{0}\left(\frac{\tau}{\left.\tau_{s 1} F_{C}-\frac{\tau}{\tau_{s 1}} \cdot a_{0-1}\right)}\right)+}\right. \\
& \frac{\left(t_{g}-t_{n}\right) \cdot\left(K_{0}+\left(K_{1}-K_{0}\right) \frac{\tau}{\tau_{s 1}}\right)}{b_{1}}\left(e^{-b_{1} F_{C}}-e^{-b_{1} \frac{\tau}{\tau_{s 1}} F_{C}}\right) \ldots \ldots .\left(0 \leq \tau \leq \tau_{s 1}\right) \\
& Q_{1-0}=c G_{0}\left(t_{g}-t_{n}\right)\left(1-e^{-b_{0} F_{C}}\right)+ \\
& \frac{\left(t_{g}-t_{n}\right) \cdot\left(K_{1}-\left(K_{1}-K_{0}\right) \frac{\tau}{\tau_{s 1}}\right)}{b_{0}}\left(e^{-b_{0} F_{C}}-e^{-b_{0} \frac{\tau}{\tau_{s 1}} F_{C}}\right)
\end{aligned}
$$




$$
\begin{gathered}
-\frac{\left(t_{g}-t_{n}\right) \cdot\left(K_{1}-\left(K_{1}-K_{0}\right) \frac{\tau}{\tau_{s 1}}\right)}{b_{1}}\left(e^{-b_{1}\left(F_{C}+\frac{\tau}{\tau_{s 1}} \cdot a_{1-0}\right)}-e^{-b_{1}\left(\frac{\tau}{\tau_{s 1}} F_{C}+\frac{\tau}{\tau_{s 1}} \cdot a_{1-0}\right)}\right) \\
\ldots \ldots .\left(0 \leq \tau \leq \tau_{s 0}\right)
\end{gathered}
$$

Where $a_{0-1}$, the max right parallel moving distance; $a_{1-0}$, the max left parallel moving distance; $b_{0}, b_{1}$ coefficient, $\left(m^{-1}\right) ; c$-mass specific heat of water. $F_{C}$-total area of radiator $\left(\mathrm{m}^{2}\right) . G_{0}$ - flux before step change. $G_{1}$ - flux after step change. $K$ - heat transfer coefficient of radiator( $\left.\mathrm{W} / \mathrm{m}^{2} .{ }^{\circ} \mathrm{C}\right) \cdot Q_{0-1}-$ dynamic heat quantity of radiator in flux largen transient $(W) \cdot Q_{1-0}-$ dynamic heat quantity of radiator in flux diminution transient $(W) . t_{n}$-average room temperature. $\left({ }^{\circ} \mathrm{C}\right)$ .$t_{g}$-feed water temperature of radiator $\left({ }^{\circ} \mathrm{C}\right) . \tau_{s 1}$ —evacuation time of the medium in the flow rate $G_{1}, \tau_{s 0}$-evacuation time of the medium in the flow rate $G_{0}$.

These two equations can be applicable not only for dynamic course, but also for steady course. Let $\tau \equiv 0$ in steady course.

\section{Dynamic Heat Measurement Error of Heat Meter in Transient}

Compared equation (3),(4) with equation (1)(or(2)) in different conditions, heat measurement error in transient can be acquired. The follow analyzing is based on the assumption that heat dissipating capacity of pipeline is too little to be considered, water supply temperature $t_{g}$ is invariable.

\subsection{Errors in Same Initial Flow Rate with Different Step Change}

The results presented in Fig.1,2. The curves showed that Eq. (1) used in transient can produce very huge errors. When system flow rate vary large from $G_{0}$ to $G_{1}$, maximum error exist in the beginning of the transient, for this moment, the feed water temperature become rising, but the backwater temperature remain unchanged, so the temperature difference rising, on the same time, system flow rate vary large, all this factors caused calculating value of heat dissipating capacity with Eq. (1) larger than Eq. (3). With the time gone, the error become small with the backwater temperature rising.

At $\tau=\tau_{S 1}$, the mass of system finish updating, backwater temperature reach its real level, the error disappeared. When system flow rate vary small from $G_{1}$ to $G_{0}$, maximum error exist in the beginning of the transient too, and it is negative value, for this moment the feed water temperature is invariable, and the backwater temperature 


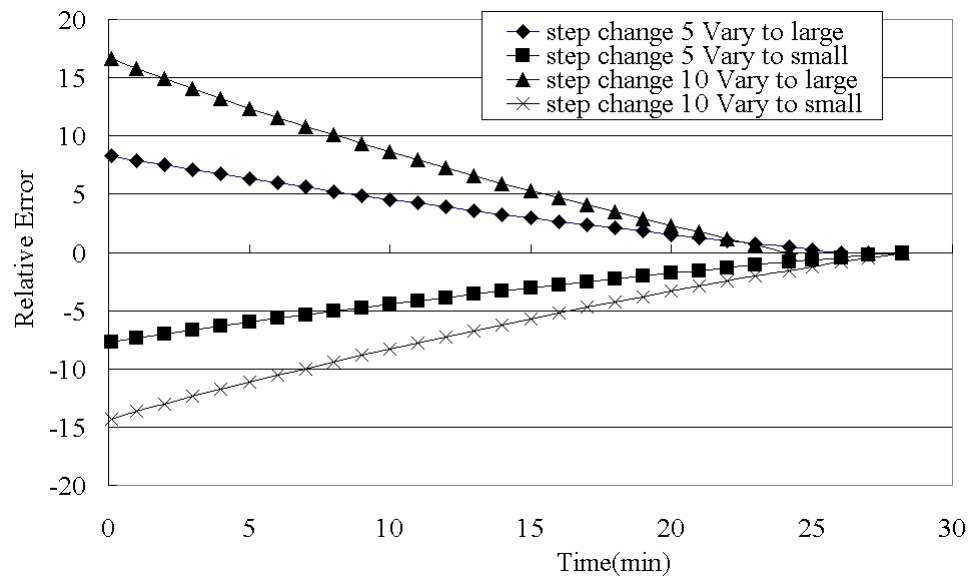

Fig. 1. Comparison of average of relative error in step change 5 and 10

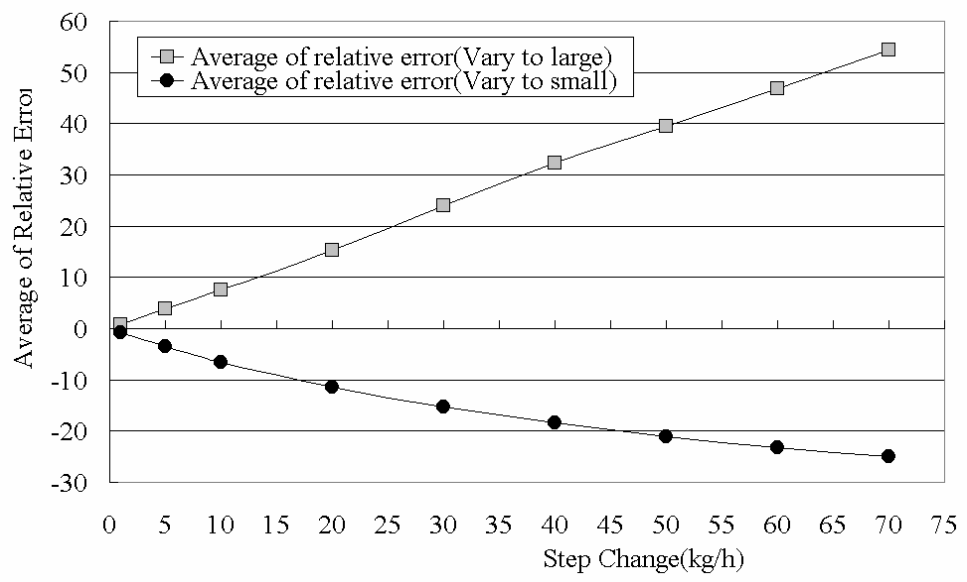

Fig. 2. Average of relative error in the same initial flow rate with different step change

also remain unchanged at the beginning, so the temperature difference is invariable, on the same time, system flow rate vary small, all this factors caused calculating value of heat dissipating capacity with Eq. (1) less than Eq.(4). With the time gone, the errors become small with the backwater temperature descending.

At $\tau=\tau_{S 0}$, the mass of system finish updating, backwater temperature reach its real level, the error disappeared. Moreover, different step change has different average of relative error, the larger step change, and the more average of relative error. Fig.1 only showed two step changes (5 and 10), more test data and comparison presented in Fig.2. 


\subsection{Errors in Same Step Change with Different Initial Flow Rate $G_{0}$}

The result showed in Fig.3 to Fig6.

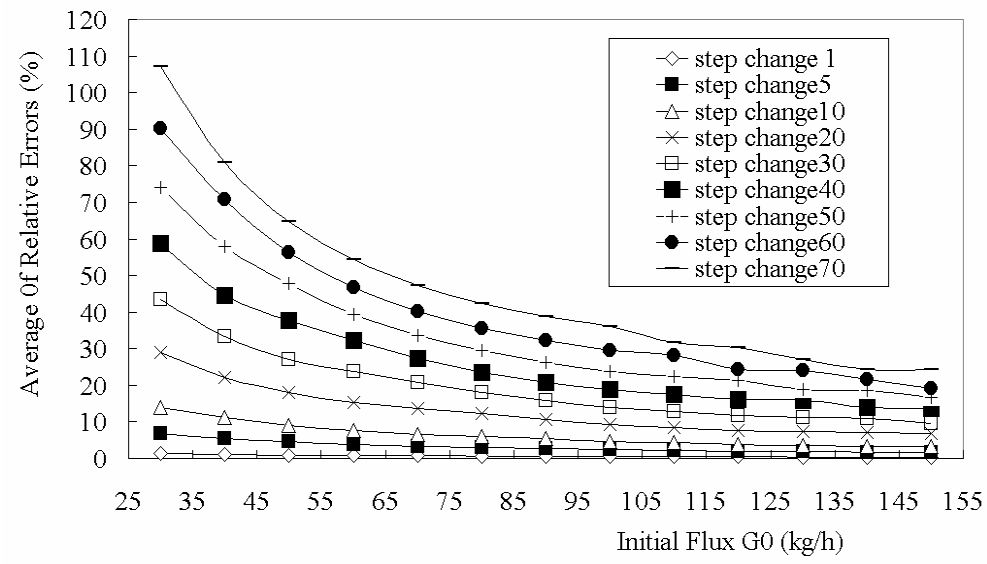

Fig. 3. Average of relative error in different initial flow rate and different step change (flow rate vary to large)

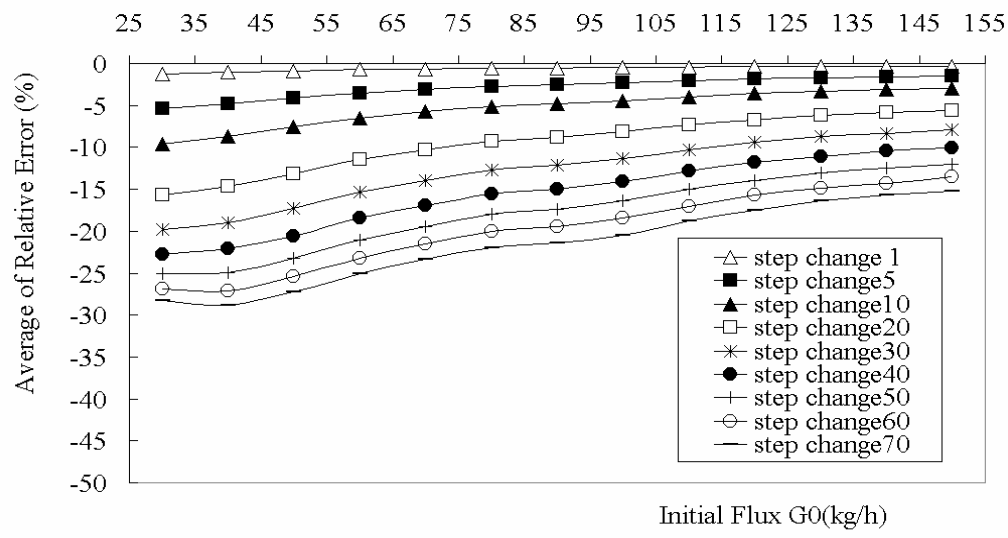

Fig. 4. Average of relative error in different initial flow rate and different step change (flow rate vary to small)

It is clear from all the results that average of relative errors increase with increasing step change. At the same step change, absolute average of relative errors is descending with the initial flow rate $G_{0}$ changing to large no matter in the transient of system flow 
rate changing to large or small. Fig.3,4 showed average of relative errors have the same trend in different step change; Average of relative error tends to unity at same step change ratio, so all data almost exhibits one line in Fig.5. In flow rate large to small transient, all data exhibits most curvature, particularly at low initial flow rate, see Fig.6. These pictures would provide some valuable reference for heat measurement function correlations. Two ways may be concerned in correcting the errors, one is to use dynamic equations in transient, the other is to use steady equation multiply a coefficient which is the function of step change ratio.

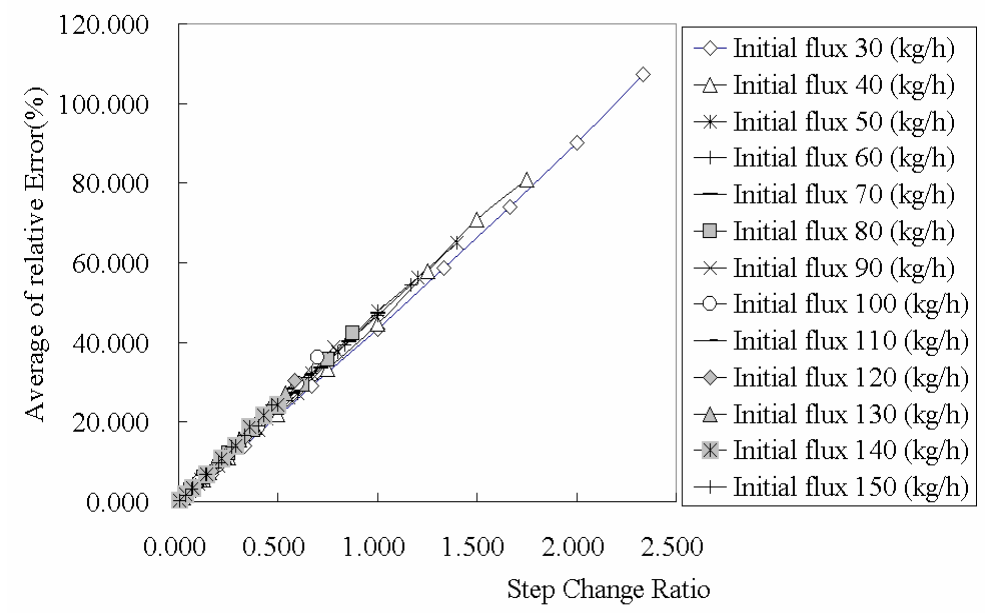

Fig. 5. Average of relative error in different step change ratio and different initial flow rate (flow rate vary to large)

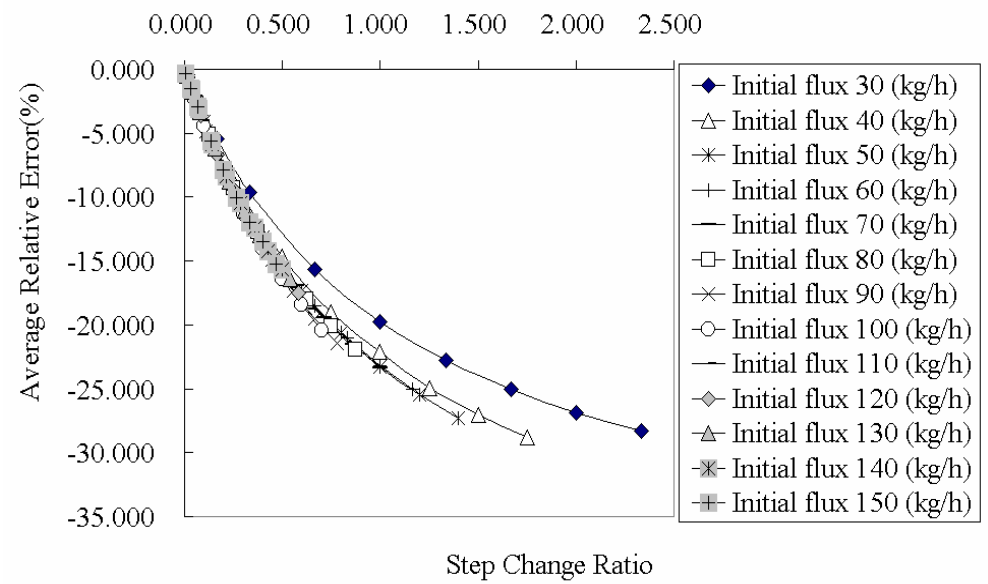

Fig. 6. Average of relative error in different step change ratio and different initial flow rate (flow rate vary to small) 


\section{Conclusion}

From discussing above, The obvious error will be produced if heat dissipating capacity of heat system is calculate by using steady-state equation (1) after step change of flow rate.

In a fixed original flow rate, As the flow rate step change is small the relative error corresponding this condition is small too, and otherwise, it is large. In a fixed step change, average relative errors decreasing with the initial flow rate increasing. These kinds of errors only exist in the transient, and a part of it can be counteracted because the sign of the relative error is reverse during the two process of flow rate increasing and decreasing. According to calculation data shown in the paper, the error can be ignored when the flow rate step change is less than $5 \mathbf{k g} / \mathbf{h}$, else it must be corrected.

The errors mentioned above only exist in transient, when system reach steady state, the errors disappear, however, the transient time is long in heating system, it is at least 30 minutes, so it is necessary to take some measures to correct them.

To correcting the errors, some program should be added in system's software, so that the heat meter can have some functions like step change discrimination, timing. In brief, heat meter should have the function to judge the quantity of the flow rate step change, so that using different formula in different working state. In brief, two ways may be concerned in correcting the errors, one is to use dynamic equations in transient, the other is to use steady equation multiply a coefficient which is the function of step change.

\section{References}

[1] Europeanstandard, EN1434:1997has the status of a DIN standard, Heatmeters,(1997)

[2] OIML-R75 International Recommendation, Heatmeters,(2002)

[3] SHOJI KUSUI and TETSUO NAGAI: An Electronic Integrating Heat Meter, IEEE TRANSACTIONS ON INSTRUMENTATION AND MEASUREMENT. VOL 39 NO S. OCTOBER,pp785-789,1990

[4] Géza Móczár,Tibor Csubák and Péter Várady: Distributed Intelligent Hierarchical System for Heat Metering and Controlling, IEEE Instrumentation and Measurement Technology Conference,Budapest,Hungary,pp2123-2128,May 21-23,2001.

[5] Géza Móczár, Tibor Csubák, and Péter Várady: Student Member, IEEE, Distributed Measurement System for Heat Metering and Control, IEEE TRANSACTIONS ON INSTRUMENTATION AND MEASUREMENT, VOL. 51, NO. 4, pp691-694, AUGUST 2002.

[6] Jin Hai-long; Pan Yong: Development and research of a new style intelligent heat meter, Chinese Journal of Sensors and Actuators vol.18, no.2 : 350-352, 2005

[7] Hao LN, Chen H, Pei HD: Development on a new household heat meter, ISTM/2005: 6TH INTERNATIONAL SYMPOSIUM ON TEST AND MEASUREMENT, VOLS 1-9, CONFERENCE PROCEEDINGS : 3090-3092, 2005

[8] Ye Xian-ming; Zhang Xiao-dong: Design on intelligent heat meter, Instrument Techniques and Sensor no.1: 10-12, 2005

[9] Fang Lide: Study on dynamic character of a new heating measurement and control system, Master's degree dissertation of Hebei University of Technology, pp18-36, 2005.3 
[10] Fang Lide, Li Xiaoting, Li Jinhai, etc: Analyze Dynamic Errors of heat meter after step change of Flow rate, Electrical Measurement \& Instrumentation (J), pp20-24, .2005.9

[11] Toma, G: Practical Test Functions Generated by Computer Algorithms, Lecture Notes Computer Science 3482 (2005), 576-585

[12] Toma, C: An Extension of the Notion of Observability at Filtering and Sampling Devices, Proceedings of the International Symposium on Signals, Circuits and Systems Iasi SCS 2001, Romania, 233--236 